\title{
Galvanic Corrosion Inhibition from Aspect of Bonding Orbital Theory: Case Study of Copper/Ruthenium Coupled Film Using 3- Pyridinecarboxylic Acid
}

\section{Kangchun Lee}

Hanyang University

\section{Seho Sun}

Hanyang University

\section{Ganggyu Lee}

Hanyang University

Gyeonghui Yoon

Hanyang University

Donghyeok Kim

Hanyang University

Junha Hwang

Hanyang University

Hojin Jeong

Hanyang University

Taeseup Song ( $\nabla$ tssong@hanyang.ac.kr)

Hanyang University

\section{Ungyu Paik}

Hanyang University

\section{Research Article}

Keywords: Copper, Electronic materials, EIS, Potentiostatic, Anodic protection, Passivation film

Posted Date: August 26th, 2021

DOI: https://doi.org/10.21203/rs.3.rs-815881/v1

License: (c) (1) This work is licensed under a Creative Commons Attribution 4.0 International License.

Read Full License 


\section{Abstract}

In this report, the galvanic corrosion inhibition between $\mathrm{Cu}$ and Ru metal films is studied, based on bonding orbital theory, using pyridinecarboxylic acid groups which show different affinities depending on the electron configuration of each metal resulting from a $\pi$-backbonding. The $\mathrm{sp}^{2}$ carbon atoms adjacent to nitrogen in the pyridine ring provide $\pi$-acceptor which forms a complex with filled d-orbital of native oxides on $\mathrm{Cu}$ and Ru metal film. The difference in the d-orbital electron density of each metal oxide leads to different $\pi$-backbonding strength, resulting in dense or sparse adsorption on native metal oxides. The dense adsorption layer is formed on native $\mathrm{Cu}$ oxide film due to the full-filled d-orbital electrons, which effectively suppresses anodic reaction in Cu film. On the other hand, only a sparse adsorption layer is formed on native Ru oxide due to its relatively weak affinity between partially filled d-orbital and pyridine groups. The adsorption behavior is investigated through interfacial interaction analysis and electrochemical interaction evaluation. Based on this finding, the galvanic corrosion behavior between $\mathrm{Cu}$ and Ru during chemical mechanical planarization (CMP) processing has been controlled.

\section{Introduction}

The contact of two metals with different electrical potentials in an electrolyte induces galvanic corrosion $[1,2]$. Especially in the semiconductor manufacturing process, the galvanic corrosion issues are constantly being pointed out as the various metals such as copper $(\mathrm{Cu})$, tantalum $(\mathrm{Ta})$, aluminum (Al), tungsten $(\mathrm{W})$, cobalt $(\mathrm{Co})$, and ruthenium ( $\mathrm{Ru}$ ) are used in interconnect, a barrier layer (liner), via filling and bottom electrode applications depending on the technology node developed. The device structure composed of these metals is constructed through chemical mechanical planarization (CMP) process followed by thin film deposition via chemical vapor deposition (CVD) and/or electroplating deposition (EPD) $[3,4]$.

As the device feature size shrinks to $5 \mathrm{~nm}$ and smaller, the $\mathrm{Ta} / \mathrm{TaN}$ material, used as the diffusion barrier metal layer with a thickness of $170 \sim 250 \AA$ in Cu interconnects, reaches a limit in the achievement of a complete Cu gap-fill and prevent electromigration (EM) of Cu (Fig. 1a) [5-8]. To overcome these limitations, Ru has been attracted much attention as a barrier layer material due to its excellent conductivity and gap-fill property. By employing Ru as a barrier layer material, the thickness of barrier metal could be reduced to $40 \sim 50 \AA$, resulting in decreased resistance-capacitance delay (Fig. 1b). It also meets integrated circuit (IC) design rule requirements of $5 \mathrm{~nm}$ and smaller. Furthermore, due to the compatibility between $\mathrm{Cu}$ and $\mathrm{Ru}$ metals, $\mathrm{Cu}$ could be deposited directly onto the Ru film via electroplating without requiring a seed layer, which is advantageous in terms of cost reduction and surface quality of the deposited film. [9-11]. For the application of the Ru metal in barrier structure, the CMP performance is required to 1:1 selectivity of removal rate on Ru and Cu metal films.

$\mathrm{Ru}$ is a chemically stable material and relatively hard compared to $\mathrm{Cu}(\mathrm{Ru}$ : Mohs 6.5 and $\mathrm{Cu}$ : Mohs 2.5). For the chemical activation of the Ru metal, potassium periodate $\left(\mathrm{KIO}_{4}\right)$ and hydrogen peroxide $\left(\mathrm{H}_{2} \mathrm{O}_{2}\right)$ 
these oxidants brings galvanic corrosion deteriorating between the $\mathrm{Cu}$ and Ru films (Fig. 1c), resulting in defects that will degrade the device performances (i.e., RC delay deterioration) consequently. In Ru barrier structure in $\mathrm{Cu}$ interconnects, $\mathrm{Cu}$ film becomes the anode (oxidation reaction as an electron donor), and Ru film acts as the cathode (reduction reaction as an electron acceptor) due to the electromotive force difference between $\mathrm{Cu}$ and Ru films $[14,15]$. Research and engineering reactions at the interface between metal films and electrolytes are crucial for reducing the electromotive force difference, especially interface resistance controlling the galvanic corrosion. In recent studies, Peethala et al. reported inhibitor substances in specific $\mathrm{KIO}_{4}$ concentrations to understand how to prevent $\mathrm{Cu}$ and Ru galvanic corrosion. The benzotriazole (BTA) and ascorbic acid (AA) can help minimize the galvanic corrosion of Cu with preferential absorption of AA on $\mathrm{Ru}$, which suppressed the cathodic reaction at $\mathrm{Ru}$ [16]. Also, Chen et al. studied that the periodate ions also formed a complex with $\mathrm{Cu}$ [17]. The reaction between periodate ions and dissolved $\mathrm{Cu}$ ions forming the $\mathrm{Cu}\left(\mathrm{IO}_{3}\right)_{2} \cdot \mathrm{nH}_{2} \mathrm{O}$ is the accelerative stage of the galvanic corrosion of the $\mathrm{Cu} / \mathrm{Ru}$ couple in $\mathrm{KIO}_{4}$ solution [18]. They suggest that adsorbed ions and chemicals act as a passivation film synergistically. At the presence of $\mathrm{K}_{2} \mathrm{MoO}_{4}$ and benzotriazole (BTA), adsorbed $\mathrm{MoO}_{4}{ }^{2-}$ ions on the metal films increase activation energy of the corrosion and form a three-dimensional network complex film with the BTAs due to the ion-dipole effect of absorbed $\mathrm{MoO}_{4}{ }^{2-}$ ions [19]. However, there is much room for research on selecting inhibitors and engineering the slurries regarding the inhibition mechanism of $\mathrm{Cu} / \mathrm{Ru}$ galvanic corrosion.

Herein, we proposed the design principle of inhibitors to minimize the galvanic corrosion at $\mathrm{Cu} / \mathrm{Ru}$ coupled films based on bonding orbital theory. The d-orbital electron densities that distinguish between native metal oxides of the $\mathrm{Cu}$ and Ru metal films cause selective adsorption affinity of the pyridine groups by $\pi$-back bonding, resulting in reduced electromotive force differences. For the analysis of selective adsorption behavior of the pyridine groups, the contact angle measurements and X-ray photoelectron spectroscopy (XPS) were conducted depending on the concentration of the pyridine group, nicotinic acid. The difference in affinity for each metal of the nicotinic acid showed a dissimilarity in the density of the inhibitor layer formation. Also, the electromotive force differences were evaluated by Tafel slope and electrochemical impedance spectroscopy (EIS) measurement. The open-circuit potential $\left(\Delta \mathrm{E}_{\mathrm{oc}}\right)$ difference between $\mathrm{Cu}$ and Ru films is $0.49 \mathrm{~V}$ conducted at a $0.05 \mathrm{M} \mathrm{KIO}_{4}$ solution with $3 \% \mathrm{H}_{2} \mathrm{O}_{2}$ at pH 10 (Table 1). A dense layer was formed on the $\mathrm{Cu}$ film in the presence of nicotinic acid, leading to the potential gap reduction between the two films from $0.49 \mathrm{~V}$ to $0.09 \mathrm{~V}$. Finally, the change in polishing removal rate and $\mathrm{Cu}$ to $\mathrm{Ru}$ selectivity were calculated through the CMP test. 
Table 1

The corrosion potentials $\left(\mathrm{E}_{\text {corr }}\right)$ and corrosion currents $\left(\mathrm{I}_{\text {corr }}\right)$ value of each $\mathrm{Cu}$ and Ru film according to inhibitor concentration.

\begin{tabular}{|lllll|}
\hline & Cu film & \multicolumn{3}{c|}{ Ru film } \\
\hline Solution system & $E_{\text {corr }}[\mathrm{V}]$ & $\mathrm{I}_{\text {corr }}\left[\mathrm{mA} / \mathrm{cm}^{2}\right]$ & $E_{\text {corr }}[\mathrm{M}]$ & $\mathrm{I}_{\text {corr }}\left[\mathrm{mA} / \mathrm{cm}^{2}\right]$ \\
\hline None & -0.27 & 0.22 & 0.22 & 0.05 \\
\hline Nicotinic acid $0.03 \mathrm{M}$ & 0.26 & 0.34 & 0.41 & 0.31 \\
\hline Nicotinic acid $0.05 \mathrm{M}$ & 0.36 & 0.47 & 0.45 & 0.42 \\
\hline
\end{tabular}

\section{Experimental Section}

Materials preparation Commercially colloidal $\mathrm{SiO}_{2}$ was used as an abrasive $\left(\mathrm{d}_{\text {mean }} \sim 70 \mathrm{~nm}\right.$, Fuso, Japan). The solid concentration of $\mathrm{SiO}_{2}$ was $5.0 \mathrm{wt} \%$. $3.0 \mathrm{wt} \%$ hydrogen peroxide $\left(\mathrm{H}_{2} \mathrm{O}_{2}\right.$, Junsei Chemical, Japan) was used as an oxidant. Nicotinic acid (Sigma Aldrich, USA) was added to the solution per solid concentration as an inhibitor. $0.01 \mathrm{M}$ manganese $(\mathbb{X})$ nitrate hydrate (Sigma Aldrich, USA) as a catalyst and $0.1 \mathrm{M}$ of citric acid (Sigma Aldrich, USA) as a chelating agent were used. $0.05 \mathrm{M}$ potassium periodate (Sigma Aldrich, USA) was used as an auxiliary oxidizer. The slurry pH was adjusted to 10.0 using potassium hydroxide (KOH, 1.0N, Daejung Chemical, Korea). 300mm electroplating Cu wafers were purchased from Advantech Korea Co., Ltd. The ruthenium wafers made through chemical vapor deposition were supplied by MEMC Korea Ltd.

Adsorption behavior analysis The adsorption behavior of inhibitor onto the $\mathrm{Cu}$ and $\mathrm{Ru}$ films was analyzed through contact angle measurements (Digidrop, GBX, Ireland) and X-ray photoelectron spectroscopy (XPS) (K-Alpha+, Thermo Fisher Scientific Messtechnik, USA). The Cu and Ru films were individually dipped in $200 \mathrm{~mL}$ of various inhibitor concentrations at $\mathrm{pH} 10.0$ for 10 minutes. Then samples were subsequently rinsed with deionized water before analysis.

Electrochemical performance investigation Potentiodynamic polarization measurement (AUT320N, Metrohm AUTOLAB, Switzerland) and electrochemical impedance spectroscopy (EIS) (AUT320N, Metrohm AUTOLAB, Switzerland) were used to characterize the electrochemical performance of $\mathrm{Cu}$ and Ru films. The counter electrode was a platinum-coated mesh, and the reference electrode was an $\mathrm{Ag} / \mathrm{AgCl}$. The size of cut samples for potentiodynamic polarization measurement and electrochemical impedance spectroscopy were $3 \times 6 \mathrm{~cm}$ (exposing $1 \mathrm{~cm}^{2}$ active area) and $1 \times 3 \mathrm{~cm}$ (exposing $1 \mathrm{~cm}^{2}$ active area), respectively (All the samples prepared in this measurement was the same as that mentioned in 2.2.). Before each experiment, the $\mathrm{Cu}$ and Ru wafer were removed from the native oxide using a buffered oxide etch (BOE) solution. The EIS was measured in a frequency range from $105 \mathrm{~Hz}$ to $10-2 \mathrm{~Hz}$, and the applied AC potential was $10-2 \mathrm{~V}_{\mathrm{rms}}$ in amplitude. 
CMP performance evaluation A coupon CMP (POLI-300, G\&P Technology, Korea) with a pad (IC 1010/Suba IV, Rohm, and Haas Electronic Materials, USA) was used for the CMP evaluation. The thickness of copper and ruthenium films was measured using a four-point probe (CMT-SR5000, Changmin Tech, Korea). The CMP evaluation to obtain material removal rates of both $\mathrm{Cu}$ and Ru films was performed three times under the 1.5 psi pressure, $79 / 80 \mathrm{rpm}$ rotation speed, and $100 \mathrm{~mL} / \mathrm{min}$ flow rate conditions.

\section{Results And Discussion}

Inhibition mechanism of pyridine functional group of nicotinic acid. - Fig. 2 represents the structure of 3pyridinecarboxylic acid (nicotinic acid) and the schematic illustration of its suggested inhibition mechanism. As shown in Fig. 2a, nicotinic acid has the formula $\mathrm{C}_{6} \mathrm{H}_{5} \mathrm{NO}_{2}$ and belongs to a group of monocarboxaylic derivatives of pyridine. Since the nitrogen atom of the pyridine functional group has electron pairs, they can form complexes by using $\sigma$-bonding (i.e., coordinate covalent bonding) with transition metal ions from oxidized metal surfaces such as $\mathrm{Cu}, \mathrm{Ir}, \mathrm{W}, \mathrm{Co}$, and Ru [20]. Such complexes mentioned above form a passivation layer on the metal film surface, preventing corrosion reactions by preventing water adsorption. However, the density of the inhibitor layer through the metal complex differs depending on the type of metal film. As shown in Fig. $2 b$, the electron configurations of metal ions in oxidized $\mathrm{Cu}$ and Ru films are $\mathrm{Cu}^{+}:(\mathrm{Ar}) 3 \mathrm{~d}^{10}$ and $\mathrm{Ru}^{4+}:(\mathrm{Kr}) 4 \mathrm{~d}^{4}$, respectively. As a result, there is a difference in terms of $\pi$-back bonding which participates in the delocalized $\pi$-electrons present in planar cyclic hydrocarbon molecules of the pyridine ring structure and filled d-orbital of metal ions (Fig. 2b) [21]. In the case of $\mathrm{Cu}$ film, the d-orbital of $\mathrm{Cu}^{+}$ion derived from $\mathrm{Cu}_{2} \mathrm{O}$ is fully occupied. Therefore, the $\pi^{-}$ electron of pyridine ring structure can form $\pi$-backbonding with the $\mathrm{Cu}^{+}$ion better than the $\mathrm{Ru}^{4+}$ ion because of the insufficient outermost electrons of the $\mathrm{Ru}^{4+}$ ion. Consequently, the affinity between nicotinic acid and $\mathrm{Cu}$ film should be more robust than its affinity with Ru film, resulting in a dense inhibitor layer formation on the surface of Cu oxide (Fig. 2c). In comparison, only a sparse inhibitor layer is formed on the Ru oxide film (Fig. 2d).

Interfacial interaction behavior analysis between $\mathrm{Cu} / \mathrm{Ru}$ films and nicotinic acid inhibitor - For analysis of interfacial interaction between $\mathrm{Cu} / \mathrm{Ru}$ films and nicotinic acid, the contact angle measurement and Xray photoelectron spectroscopy (XPS) are evaluated. The contact angle measurements are a valuable tool for measuring the hydrophobicity of thin films. In general, the nitrogen atom from the pyridine functional group of nicotinic acid forms a complex by creating a $\sigma$-bond between its electron pair and metal ions from oxidized metal films. It forms an adsorption layer and makes a hydrophobic surface [22]. The contact angle values of $\mathrm{Cu}$ and Ru films as a function of nicotinic acid concentrations were shown in Fig. $3 \mathrm{a}$ and $3 \mathrm{~b}$. The contact angle values of $\mathrm{Cu}$ and $\mathrm{Ru}$ immersed in a slurry without nicotinic acid were $28.7^{\circ}$ and $58.4^{\circ}$, respectively. The difference in the contact angle value means that the intrinsic surface propertv of Ru film is more hvdrophobic than the Cu film. With the addition of nicotinic acid, the contact Loading [MathJax]/jax/output/CommonHTML/fonts/TeX/fontdata.js 
angle values of $\mathrm{Cu}$ and Ru films increased to $41.9^{\circ}$ and $62.1^{\circ}$, respectively. The increase of contact angle values (i.e., increased hydrophobicity) of both $\mathrm{Cu}$ and Ru films with nicotinic acid-treated is commonly attributed to a specific orientation of adsorbed nicotinic acid molecules hydrophobic pyridine group to form a protective hydrophobic layer. The change in contact angle value with and without nicotinic acid was $13.2^{\circ}$ for $\mathrm{Cu}$ and $3.7^{\circ}$ for $\mathrm{Ru}$, and the amount of contact angle change is large for $\mathrm{Cu}$ film. It means that nicotinic acid adsorbed more onto the Cu film than the Ru film.

The XPS analysis was utilized to characterize the chemical composition changes of $\mathrm{Cu}$ and Ru films after being immersed for $10 \mathrm{~min}$ in different slurries (Fig. 3c-f). Figure $3 \mathrm{c}$ and Fig. 3d show the exemplary spectra of $\mathrm{Cu}$ film; $\mathrm{Cu} 2 \mathrm{p}$ and $\mathrm{Cu} 01$ s, respectively. In Fig. $3 c$, the metallic $\mathrm{Cu}$ binding energy for $2 p^{3 / 2}$ is located at $932.2 \mathrm{eV}$. The $\mathrm{Cu}$ binding energies for $\mathrm{CuO}$ and $\mathrm{Cu}_{2} \mathrm{O}$ are found at $933.8 \mathrm{eV}$ and $932.0 \mathrm{eV}$, respectively. These results are consistent with the other research reports [23-26]. The $\mathrm{Cu}$ and $\mathrm{Cu}_{2} \mathrm{O}$ peak intensities were significantly decreased according to the concentration of the nicotinic acid. At the addition of the $0.03 \mathrm{M}$ nicotinic acid, there was no significant change in $\mathrm{Cu} 2 \mathrm{p}$ peak intensity. However, it was confirmed that when the concentration was increased to $0.05 \mathrm{M}$, the Cu2p peak intensity was overall lowered. The decrease in $\mathrm{Cu} 2 \mathrm{p}$ peak intensity is that nicotinic acid adsorbed onto the $\mathrm{Cu}_{2} \mathrm{O}$, forming a dense inhibitor layer.

Meanwhile, the binding energy peaks for $\mathrm{CuO}$ and $\mathrm{Cu}_{2} \mathrm{O}$ in the $\mathrm{Cu} 01 \mathrm{~s}$ spectra are detected at $534.0 \mathrm{eV}$ and $529.7 \mathrm{eV}$, respectively. The $\mathrm{Cu} 01 \mathrm{~s}$ peak from Fig. $3 \mathrm{~d}$ also shows that the $\mathrm{Cu}_{2} \mathrm{O}$ peak rapidly decreases with the nicotinic acid addition. This suggests that the pyridine functional group of nicotinic acid adsorbs well to $\mathrm{Cu}_{2} \mathrm{O}\left(\mathrm{Cu}^{+}\right.$state, fully occupied d-orbital) rather than $\mathrm{CuO}\left(\mathrm{Cu}^{2+}\right.$ state, partially occupied d-orbital).

Figure $3 e$ and $3 f$ show the fine spectra of Ru film; Ru $3 d$ and Ru 01 s, respectively. The metallic Ru binding energies for $3 d^{5 / 2}$ and $3 d^{3 / 2}$ are located at $280.0 \mathrm{eV}$ and $284.4 \mathrm{eV}[27,28]$. Ru binding energy for $\mathrm{RuO}_{2}$ is found to be $280.8 \mathrm{eV}$ and $285.0 \mathrm{eV}$. Because $\mathrm{RuO}_{2} \bullet \mathrm{H}_{2} \mathrm{O}$ is a metallic oxide with a partially filled conduction band, the core-hole coupling may occur on this surface [28]. Thus, $\mathrm{RuO}_{3}$ is considered present with the bulk phase of $\mathrm{RuO}_{2}$, and the binding energy peaks at $282.3 \mathrm{eV}$ and $286.5 \mathrm{eV}$, respectively. Meanwhile, the binding energy peaks for $\mathrm{RuO}_{2}$ and $\mathrm{RuO}_{3}$ appear at $529.2 \mathrm{eV}$ and $530.7 \mathrm{eV}$ in Ru 01s spectra [29, 30]. No change with nicotinic acid concentration was observed in the case of both Ru 3d (Fig. 3e) and Ru 01s (Fig. 3f) XPS results. In other words, the above results mean that nicotinic acid was barely adsorbed on the Ru film. These results correspond with the contact angle measurements.

Electrochemical interaction evaluation between $\mathrm{Cu} / \mathrm{Ru}$ films and nicotinic acid inhibitor. - For analysis on electrochemical properties, the potentiodynamic plots and Nyquist plots are evaluated. The corrosion potentials $\left(\mathrm{E}_{\text {corr }}\right)$ and corrosion currents $\left(\mathrm{I}_{\text {corr }}\right)$ of the $\mathrm{Cu}$ and Ru films under the different nicotinic acid concentrations are noted in Fig. 4a-c and Table 1. As shown in Fig. 4a-c, the Tafel curves of Cu and Ru films were evaluated throuah potentiodvnamic polarization measurements. The potential difference Loading [MathJax]/jax/output/CommonHTML/fonts/TeX/fontdata.js 
between $\mathrm{Cu}$ and Ru films decreased from $0.49 \mathrm{~V}$ to $0.09 \mathrm{~V}$ as inhibitor concentrations increased from $0 \mathrm{M}$ to $0.05 \mathrm{M}$. The galvanic corrosion occurs in the heterojunction of $\mathrm{Cu} / \mathrm{Ru}$ films because two metal films have different potentials. In Cu film, oxidation reactions that donate electrons occur (as an anodic site), while reduction reactions occur in the Ru film that accepts electrons (as a cathodic site). Therefore, by controlling the potential of both films by reducing the gap of potential difference, the redox reaction can be suppressed, resulting in galvanic corrosion prevention. The experimental results in Fig. 4c show that the potential difference between $\mathrm{Cu}$ and $\mathrm{Ru}$ is significantly reduced to $0.09 \mathrm{~V}$, indicating the galvanic corrosion between $\mathrm{Cu}$ and Ru films was suppressed. On the other hand, the $\mathrm{Cu}$ film potential change is $0.63 \mathrm{~V}$ (from $-0.27 \mathrm{~V}$ to $0.36 \mathrm{~V}$ ), more significant than that of Ru film $(0.23 \mathrm{~V}$, from $0.22 \mathrm{~V}$ to $0.45 \mathrm{~V}$ ). In both cases, adsorption of nicotinic acid tended to form an inhibitor passivation layer leading to the potential value increase. Still, the $\mathrm{E}_{\text {corr }}$ value change of $\mathrm{Cu}$ film was more extensive than that of Ru film. This phenomenon is due to the denser layer formation on the Cu film, consistent with the adsorption affinity trends of nicotinic acid mentioned above. EIS (Electrochemical impedance spectroscopy) was performed to evaluate the barrier protection properties of nicotinic acid to both $\mathrm{Cu}$ and $\mathrm{Ru}$ films. The impedance data were fitted using the electrical equivalent circuits with three resistances and two constant phase elements (CPE) shown in Fig. S1. $R_{S}$ represents the solution resistance, and $R_{f}$ is the film resistance. $R_{1}$ includes the $R_{c t}$ (charge transfer resistance), $R_{d}$ (diffusion layer resistance), and $R_{a}$ (accumulation resistance) at the metal/solution interface [31, 32]. The $\mathrm{CPE}_{1}$ and $\mathrm{CPE}_{2}$ represent the film capacitance and electric doublelayer capacitance, respectively. From Fig. $S 1$, the $R_{p}$ (polarization resistance) values, representing the corrosion inhibition effect characteristics, consisted of $R_{f}$ and $R_{1}$. Therefore, obtaining a high $R_{p}$ value indicates an improved inhibition effect. In Table 2 , the $R_{p}$ value change is more considerable for Cu film because nicotinic acid adsorbed more onto $\mathrm{Cu}$ film. That is consistent with the adsorption behaviors and potentiodynamic polarization measurements described earlier.

Table 2

Polarization resistance $\left(R_{p}\right)$ and inhibition efficiency $(\eta)$ of metal films according to inhibitor concentration.

\begin{tabular}{|lllll|}
\hline & Cu film & \multicolumn{3}{l|}{ Ru film } \\
\hline Solution system & $\mathbf{R}_{\mathrm{p}}\left[\Omega \cdot \mathrm{cm}^{2}\right]$ & $\eta[\%]$ & $\mathbf{R}_{\mathrm{p}}\left[\Omega \cdot \mathrm{cm}^{2}\right]$ & $\eta[\%]$ \\
\hline None & 5704.2 & - & 69518 & - \\
\hline Nicotinic acid 0.03M & 13830 & 58.75 & 74011 & 6.07 \\
\hline Nicotinic acid 0.05M & 49897 & 88.57 & 78549 & 11.50 \\
\hline
\end{tabular}


Nyquist plots as a function of nicotinic acid concentrations for $\mathrm{Cu}$ and $\mathrm{Ru}$ films at $\mathrm{pH} 10$ are shown in Fig. $4 \mathrm{~d}$ and Fig. 4e, respectively. As nicotinic acid concentrations increase, the real impedance difference at lower and higher frequencies for both films was increased, leading to an increase in $R_{p}$ value (Table 2). That is owing to the formation of the inhibitor protection layer on each film surface. Detailed impedance parameters and inhibition efficiencies $(\eta \%)$ are listed in Table 2. The inhibition efficiency could be calculated from the polarization resistance values as follows:

$$
\eta[\backslash \%]=\frac{R_{p}-R_{p}^{0}}{R_{p}} \times 100
$$

(where, $R_{p}^{0}$ is the polarization resistance without nicotinic acid.) The increase in the inhibition effect (i.e., increasing $R_{p}$ value) due to nicotinic acid adsorption is more dramatic in Cu film (Fig. $4 \mathrm{~d}$ and Fig. S2) comparing with Ru film (Fig. 4e and Fig. S3).

Effect of pyridine functional group on $\mathrm{Cu}$ and Ru removal rate and surface roughness. - Fig. 5a and Table 3 represent the removal rates of $\mathrm{Cu}$ and $\mathrm{Ru}$ films under the different inhibitor conditions and their removal selectivity at $\mathrm{pH} 10$ by the CMP process. Before the CMP process, the colloidal stability of each slurry is observed by zeta potential analysis (Fig. S4) and large particle counter evaluation (Fig. S5), which shows no harmful effects on the colloidal stability as CMP slurries. Without nicotinic acid as an inhibitor, the initial removal rates of $\mathrm{Cu}$ and Ru film were $95.98 \AA \AA 30$ s and $24.85 \AA / 30$ s, respectively, with a $\mathrm{Cu}$ to Ru selectivity of 3.86. However, as nicotinic acid content increased, the removal rate of Cu film decreased steeply from $95.98 \AA \AA$ /30s to $26.23 \AA ̊ / 30$ s. In contrast, the removal rate of Ru film was maintained at a constant value within the margin of error range regardless of the nicotinic acid concentration (Ultimately optimized with the Cu to Ru selectivity of $1: 1$ ). The affinity between nicotinic acid and Ru film is relatively smaller than that of Cu film, confirmed by XPS and contact angle results above. Therefore, the amount of nicotinic acid adsorbed on Ru film is comparatively weak and insignificant. This result is consistent with the small potential change $\left(\Delta \mathrm{E}_{\text {corr }}: 0.23 \mathrm{~V}\right)$ observed by the potentiodynamic polarization measurements. In a sub-5nm logic semiconductor device using a ruthenium barrier structure, the $\mathrm{Cu}$ to Ru selectivity requirement to achieve a completely flat surface is $1: 1$ for the Ru barrier CMP $[12,19]$. That is to minimize defects such as dishing, erosion and protrusion. Therefore, using nicotinic acid as an inhibitor with an affinity difference between $\mathrm{Cu}$ and Ru films prevents galvanic corrosion and controls the $\mathrm{Cu}$ to Ru selectivity simultaneously. 
Table 3

Results of the removal rate and selectivity between $\mathrm{Cu} / \mathrm{Ru}$ films according to inhibitor concentration.

\begin{tabular}{|llll|}
\hline & $\begin{array}{l}\text { Cu removal rate } \\
{[\AA / 30 s]}\end{array}$ & $\begin{array}{l}\text { Ru removal rate } \\
{[\AA / 30 s]}\end{array}$ & $\begin{array}{l}\text { Selectivity } \\
{[\mathrm{Cu} / \mathrm{Ru}]}\end{array}$ \\
\hline None & 95.98 & 24.85 & 3.86 \\
\hline Nicotinic acid $0.03 \mathrm{M}$ & 41.33 & 24.52 & 1.69 \\
\hline Nicotinic acid $0.05 \mathrm{M}$ & 26.23 & 25.0 & 1.05 \\
\hline
\end{tabular}

Additionally, as shown in Fig. 5b, the RMS (Root-mean-square) value of surface roughness $\left(R_{\mathrm{q}}\right)$ was estimated using AFM (Atomic Force Microscope) for each sample of $\mathrm{Cu}$ and Ru. Since Ru is a chemically more inert material compare to $\mathrm{Cu}$, the $\mathrm{R}_{\mathrm{q}}$ value of $\mathrm{Ru}$ is smaller than that of $\mathrm{Cu}$ in all cases regardless of the nicotinic acid concentration. Meanwhile, as the concentration of nicotinic acid increased, the $\mathrm{R}_{\mathrm{q}}$ value of $\mathrm{Cu}$ gradually decreased. This indicates that a smooth surface with improved roughness was obtained because the dissolution rate of Cu film was suppressed by forming a dense inhibiting layer. On the other hand, the improvement of Ru roughness is much smaller than that of the $\mathrm{Cu}$ film due to the suggested sparse inhibiting layer formation on Ru film.

\section{Conclusions}

The pyridine functional group inhibiting mechanism of nicotinic acid for $\mathrm{Cu}$ and Ru films was thoroughly investigated. Based on the results from adsorption affinity experiments (i.e., contact angle measurement and XPS) and electrochemical performances (i.e., potentiodynamic polarization measurement and EIS), we came to the following conclusions:

1. The difference in adsorption affinity of the pyridine functional group for $\mathrm{Cu}$ and $\mathrm{Ru}$ films is attributed to the difference in the electron configuration between $\mathrm{Cu}^{+}$ions and $\mathrm{Ru}^{4+}$ ions.

2. Nicotinic acid with a pyridine ring structure has a better affinity for $\mathrm{Cu}$ film than Ru film because it forms both $\sigma$-bonds and $\pi$-back bonds.

3. Therefore, a denser inhibitor layer is formed on the copper oxide from $\mathrm{Cu}$ film, which reduces the anodic reactions.

4. The increased electrical potential in Cu film due to the dense inhibitor layer can significantly reduce its potential gap compared to Ru film. That leads to suppressing galvanic corrosion for $\mathrm{Cu} / \mathrm{Ru}$ couples.

5. Furthermore, the dense inhibitor layer reduces the Cu removal rate by forming a passivation bed. As a result, almost 1:1 selectivity for copper to ruthenium could be achieved in the Ru barrier structure during the CMP process.

\section{Declarations}




\section{Acknowledgments}

This work was supported by the Human Resources Program in Energy Technology of the Korea Institute of Energy Technology Evaluation and Planning (KETEP), which was granted financial resources from the Ministry of Trade, Industry \& Energy, Republic of Korea (20194010201890 \& 20194030202450)

\section{References}

1. Sun, C. et al. Visualization of electrochemical behavior in carbon steel assisted by machine learning,Applied Surface Science, (2021)150412.

2. Yang, G. L. et al. Nickel interlayer enables indirect corrosion protection of magnesium alloy by photoelectrochemical cathodic protection. Appl. Surf. Sci, 558, 149840 (2021).

3. Krishnan, M., Nalaskowski, J. W. \& Cook, L. M. Chemical mechanical planarization: slurry chemistry, materials, and mechanisms. Chemical reviews, 110, 178-204 (2010).

4. Zhang, Z. et al. A novel approach of chemical mechanical polishing for cadmium zinc telluride wafers. Scientific reports, 6, 1-7 (2016).

5. Lane, M., Liniger, E. \& Lloyd, J. R. Relationship between interfacial adhesion and electromigration in Cu metallization. Journal of Applied Physics, 93, 1417-1421 (2003).

6. Yang, C. C. et al. Characterization of "Ultrathin-Cu"/Ru (Ta)/TaN liner stack for copper interconnects. IEEE Electron Device Letters, 31, 722-724 (2010).

7. Nogami, T. et al. CVD Co and its application to Cu damascene interconnections, in: 2010 IEEE International Interconnect Technology Conference, IEEE 2010, pp. 1-3.

8. Zhou, J. et al. Surface action mechanism and planarization effect of sarcosine as an auxiliary complexing agent in copper film chemical mechanical polishing. Appl. Surf. Sci, 529, 147109 (2020).

9. Peethala, B., Roy, D. \& Babu, S. Controlling the galvanic corrosion of copper during chemical mechanical planarization of ruthenium barrier films. Electrochemical and Solid-State Letters, 14, H306-H310 (2011).

10. Huang, Q. et al. Enhanced grain growth of electroplated copper on cobalt-containing seed layer. Journal of The Electrochemical Society, 160, D3045-D3050 (2013).

11. Lin, J. Y., Chou, S. W. \& Cheng, M. Y. Investigation of agglomerated Cu seed on Cu oxidation after chemical mechanical planarization. Applied surface science, 257, 547-552 (2010).

12. Peethala, B. \& Babu, S. Ruthenium polishing using potassium periodate as the oxidizer and silica abrasives. Journal of The Electrochemical Society, 158, H271-H276 (2011).

13. Cheng, J., Wang, T., Jiang, L. \& Lu, X. Surface characteristics of ruthenium in periodate-based slurry during chemical mechanical polishing. Appl. Surf. Sci, 351, 401-409 (2015).

14. Yu, K. et al. Study of Cu bimetallic corrosion in CMP chemical environments using optical scanning and micropattern corrosion screening. ECS Transactions, 35, 173-184 (2011). 
15. Cheng, J., Wang, T. \& Lu, X. Galvanic corrosion inhibitors for $\mathrm{Cu} / \mathrm{Ru}$ couple during chemical mechanical polishing of Ru. ECS Journal of Solid State Science and Technology, 6, 62 (2016).

16. Peethala, B., Roy, D. \& Babu, S. Controlling the galvanic corrosion of copper during chemical mechanical planarization of ruthenium barrier films, Electrochemical and solid state letters, 14 (2011)H306.

17. Cheng, J., Wang, T., He, Y. \& Lu, X. Material removal mechanism of copper chemical mechanical polishing in a periodate-based slurry. Appl. Surf. Sci, 337, 130-137 (2015).

18. Cheng, J., Pan, J., Wang, T. \& Lu, X. Micro-galvanic corrosion of $\mathrm{Cu} / \mathrm{Ru}$ couple in potassium periodate (KIO4) solution. Corros. Sci, 137, 184-193 (2018).

19. Cheng, J., Wang, T., Mei, H., Zhou, W. \& Lu, X. Synergetic effect of potassium molybdate and benzotriazole on the CMP of ruthenium and copper in KIO4-based slurry. Applied surface science, 320, 531-537 (2014).

20. Bleeke, J. R. \& Metallabenzenes Chemical reviews, 101, 1205-1228 (2001).

21. Huang, X., Zhai, H. J., Kiran, B. \& Wang, L. S. Observation of d-orbital aromaticity. Angew. Chem. Int. $E d, 44,7251-7254$ (2005).

22. Borowiak-Resterna, A., Klonowska, K., Olszanowski, A. \& Tomaszewska, M. Photostability of hydrophobic amides of pyridinecarboxylic acid as copper extractants from chloride media. Journal of Photochemistry and Photobiology A: Chemistry, 185, 181-187 (2007).

23. Ertl, G., Hierl, R., Knözinger, H., Thiele, N. \& Urbach, H. XPS study of copper aluminate catalysts. Applications of Surface Science, 5, 49-64 (1980).

24. Mclntyre, N. \& Cook, M. X-ray photoelectron studies on some oxides and hydroxides of cobalt, nickel, and copper, Analytical chemistry, 47 (1975)2208-2213.

25. Deroubaix, G. \& Marcus, P. X-ray photoelectron spectroscopy analysis of copper and zinc oxides and sulphides. Surface and Interface Analysis, 18, 39-46 (1992).

26. Robert, T., Bartel, M. \& Offergeld, G. Characterization of oxygen species adsorbed on copper and nickel oxides by X-ray photoelectron spectroscopy. Surf. Sci, 33, 123-130 (1972).

27. Walker, R. Triazole, benzotriazole and naphthotriazole as corrosion inhibitors for copper. Corrosion, 31, 97-100 (1975).

28. Wang, Z. et al. Controlling of Ru/Cu Removal Rate Selectivity during CMP by Using Ammonium Sulfate and Inhibitor. ECS Journal of Solid State Science and Technology, 8, 509-515 (2019).

29. Kim, I. K., Cho, B. G., Park, J. G., Park, J. Y. \& Park, H. S. Effect of pH in Ru slurry with sodium periodate on Ru CMP. Journal of The Electrochemical Society, 156, H188-H192 (2009).

30. Blouin, M. \& Guay, D. Activation of ruthenium oxide, iridium oxide, and mixed Ruxlr1 - x oxide electrodes during cathodic polarization and hydrogen evolution. Journal of the electrochemical society, 144, 573-581 (1997).

31. Özcan, M., Dehri, I. \& Erbil, M. Organic sulphur-containing compounds as corrosion inhibitors for mild ctepl in aridir media correlation hetween inhihition efficiency and chemical structure. Applied Loading [MathJax]/jax/output/CommonHTML/fonts/TeX/fontdata.js 
surface science, 236, 155-164 (2004).

32. Lgamri, A., El Makarim, H. A., Guenbour, A., Bachir, A. B. \& Aries, L. S. El Hajjaji, Electrochemical study of the corrosion behaviour of iron in presence of new inhibitor in $1 \mathrm{M} \mathrm{HCl}$, Progress in organic coatings, 48 (2003)63-70.

\section{Figures}

(a)

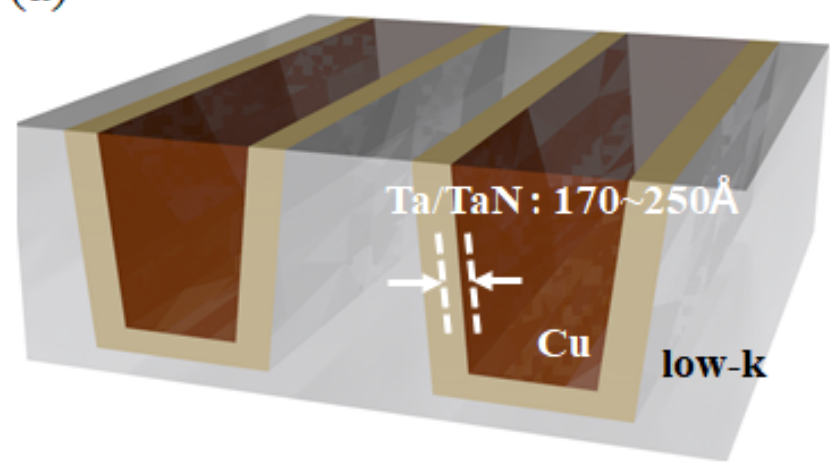

(b)
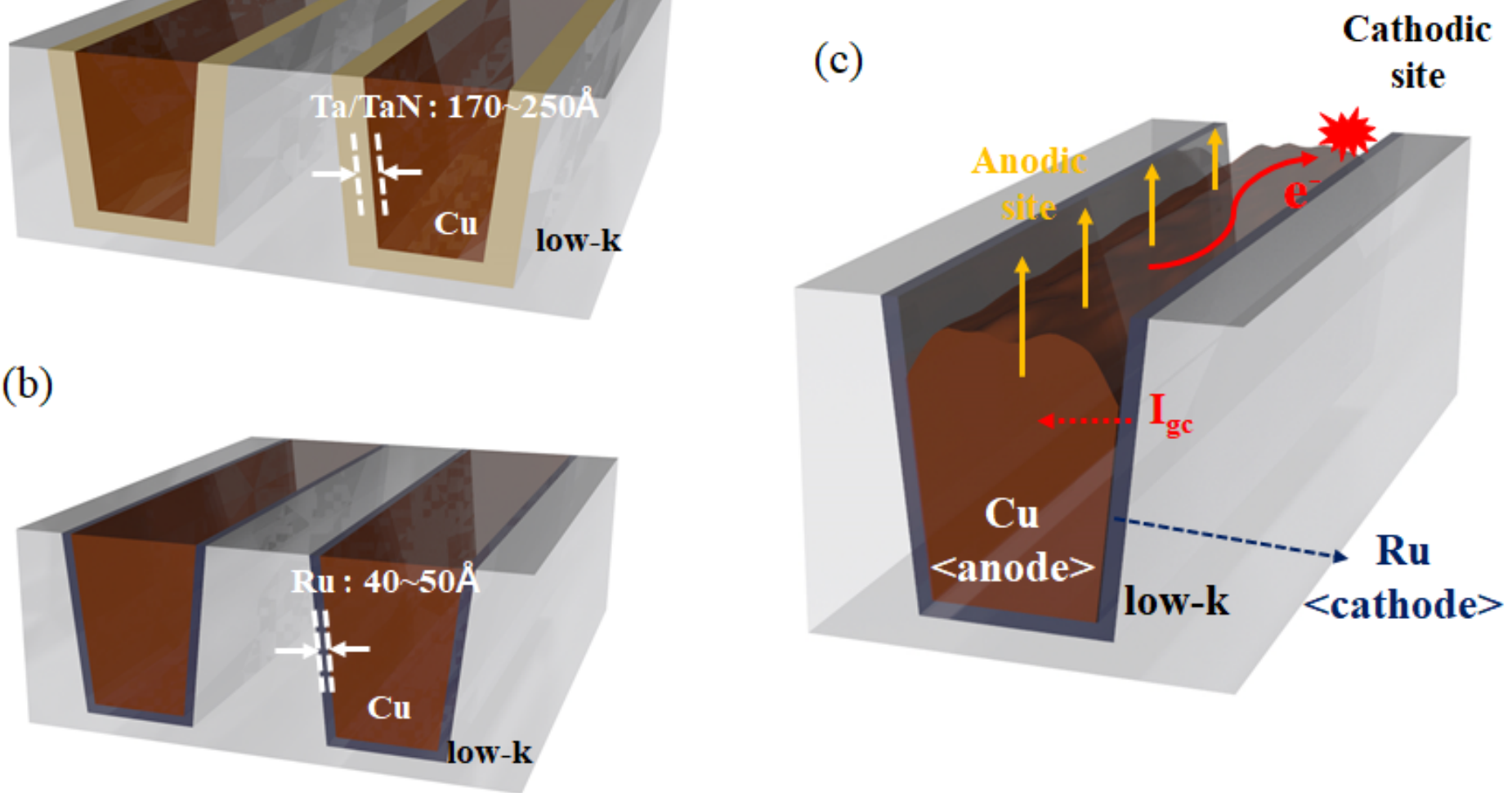

\section{Figure 1}

Schematic illustration of (a) Ta/TaN barrier structure, (b) Ru barrier structure, and (c) Galvanic corrosion in a $\mathrm{Cu} / \mathrm{Ru}$ bimetallic system; for $\mathrm{Cu}$ interconnect in back-end-of-the-line (BEOL) process. 
(a)

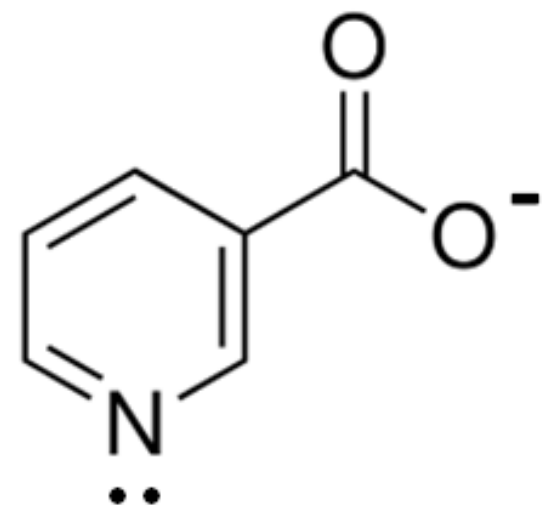

(c)

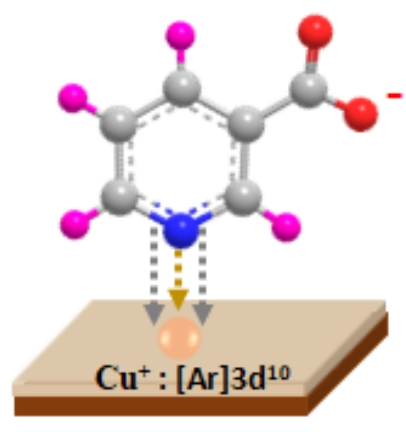

Dense inhibitor layer formation

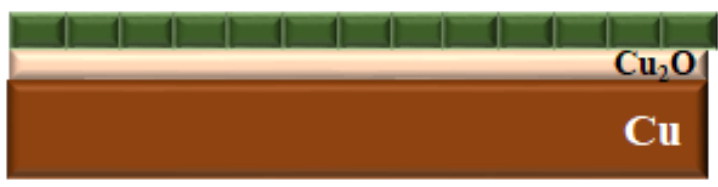

(b)

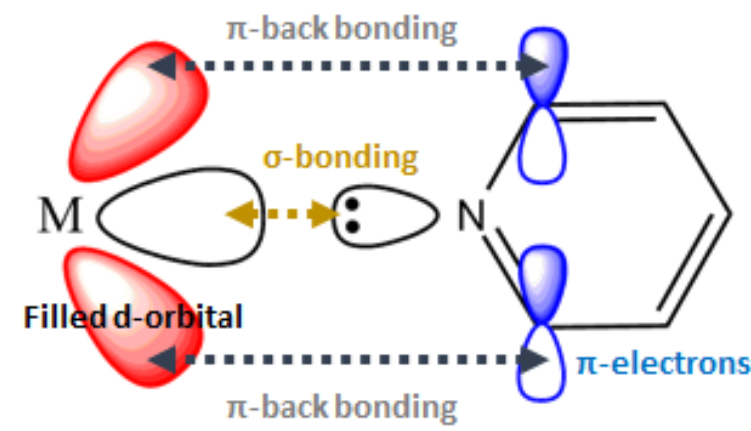

$$
\begin{aligned}
& { }^{29} \mathrm{Cu}:[\mathrm{Ar}] 3 \mathrm{~d}^{10} 4 \mathrm{~s}^{1} \rightarrow \underline{\mathrm{Cu}^{+}:[\mathrm{Ar}] 3 \mathrm{~d}^{10}} \\
& { }^{44} \mathrm{Ru}:[\mathrm{Kr}] 4 \mathrm{~d}^{7} 5 \mathrm{~s}^{1} \rightarrow \underline{\mathrm{Ru}^{4+}:[\mathrm{Kr}] 4 \mathrm{~d}^{4}}
\end{aligned}
$$

(d)
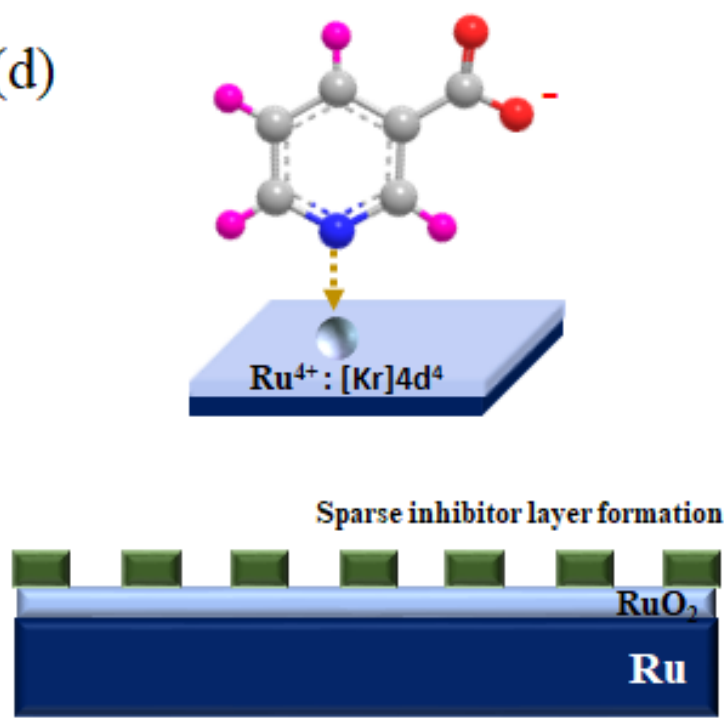

Figure 2

(a) Structure of nicotinic acid. (b) Formation of $\sigma$-bonding and $\pi$-back bonding between pyridine functional group and metal ion. The inhibition mechanism of nicotinic acid on (c) Cu blanket wafer and (d) Ru blanket wafer 

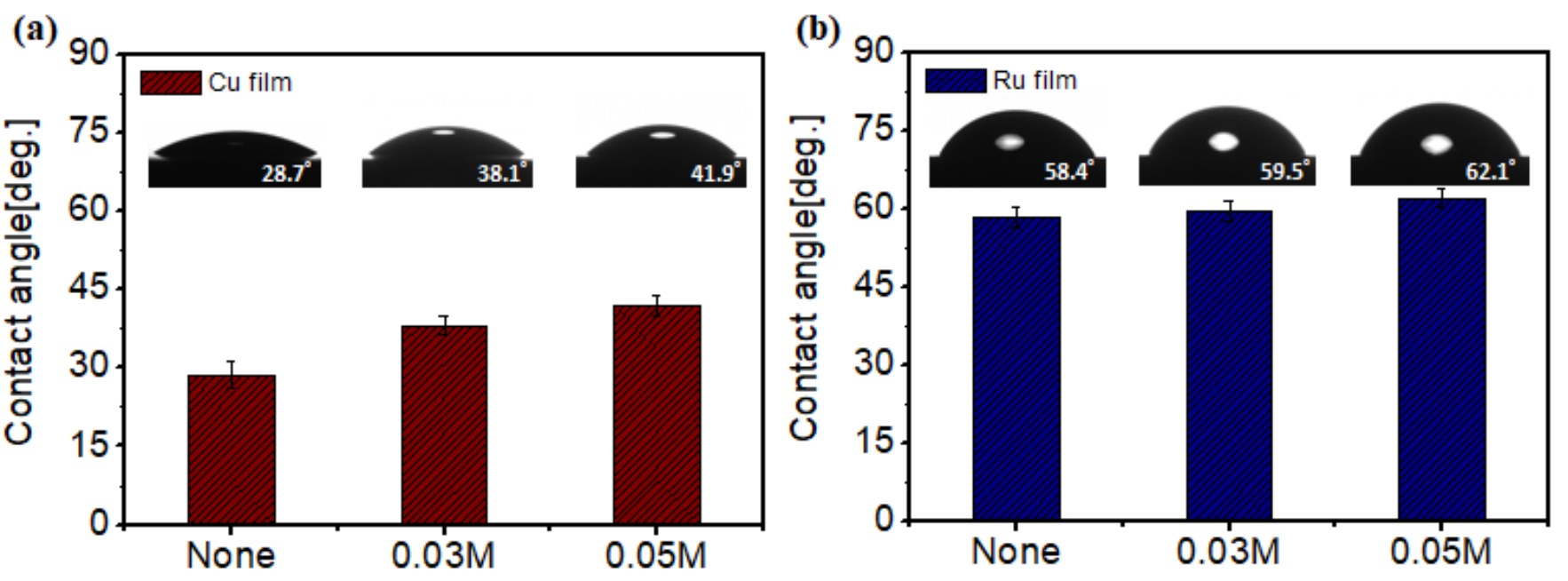

(c) $\mathrm{Cu} 2 \mathrm{p}$
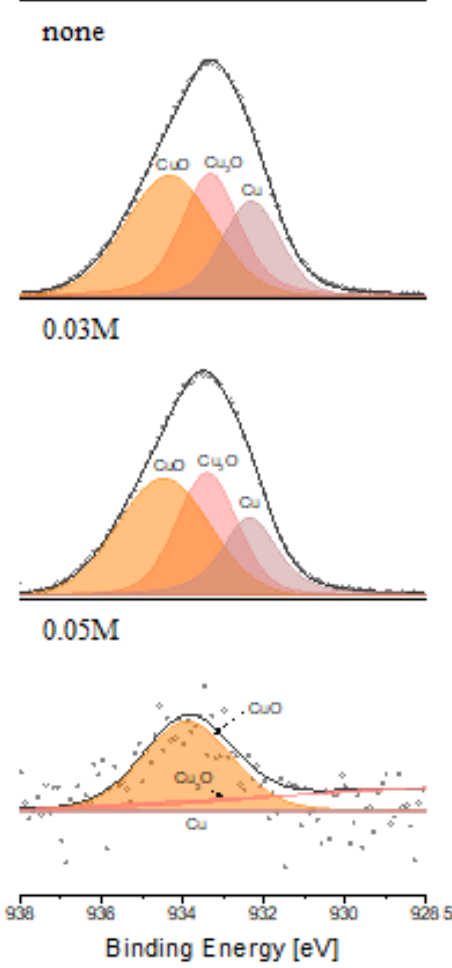

(d) $\mathrm{Cu} \mathrm{O} \mathrm{1s}$
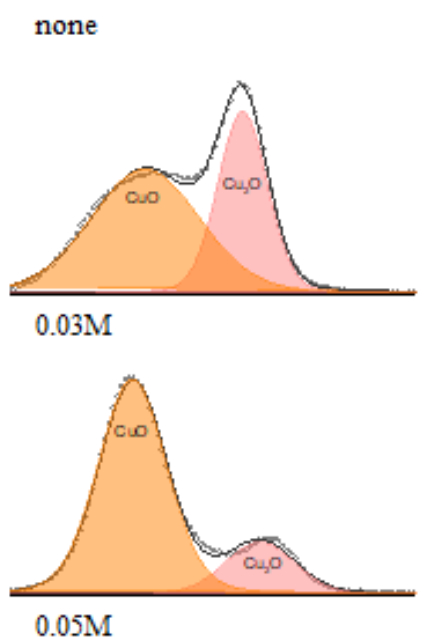

(e) Ru 3d

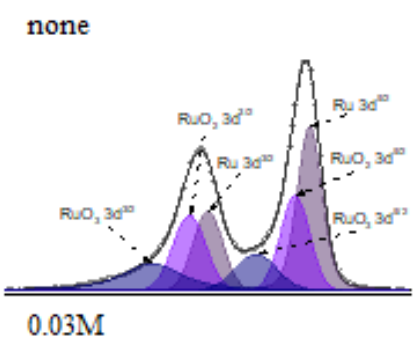

(f) Ru O 1s
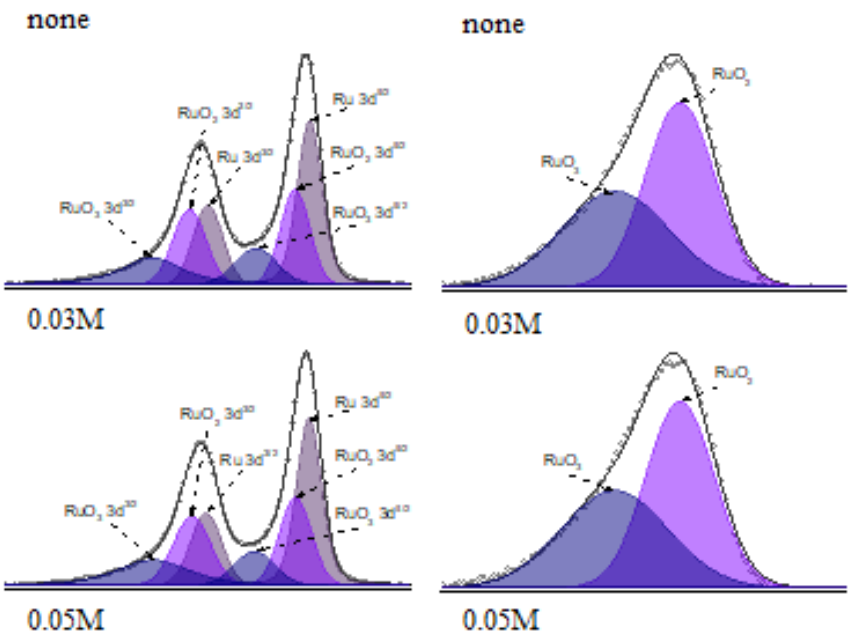
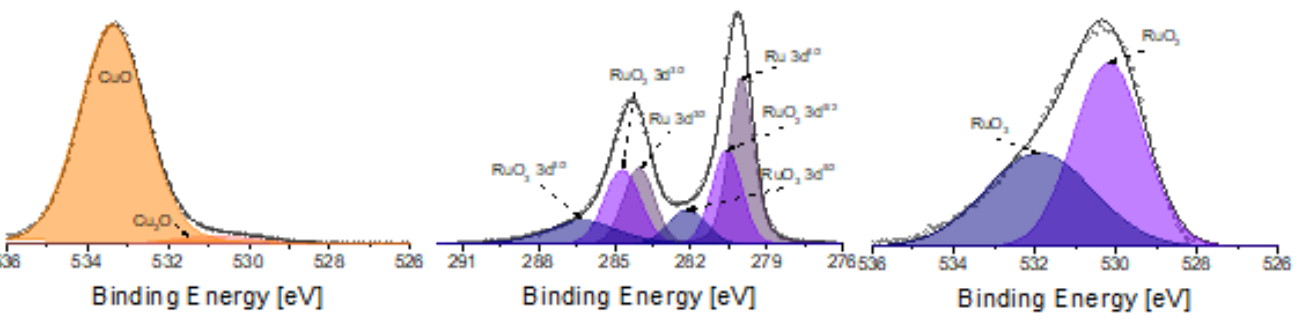

\section{Figure 3}

Interfacial interaction behavior analysis between $\mathrm{Cu} / \mathrm{Ru}$ films and nicotinic acid inhibitor. (a-b) Contact angle measurement of $\mathrm{Cu}$ and Ru films after dipping as a function of inhibitor concentrations at $\mathrm{pH} 10$. (c-f) X-ray photoelectron spectroscopy (XPS) results for (c) Cu 2p, (d) Cu 01s, (e) Ru 3d and (f) Ru 01s as a function of inhibitor concentrations at $\mathrm{pH} 10$. 

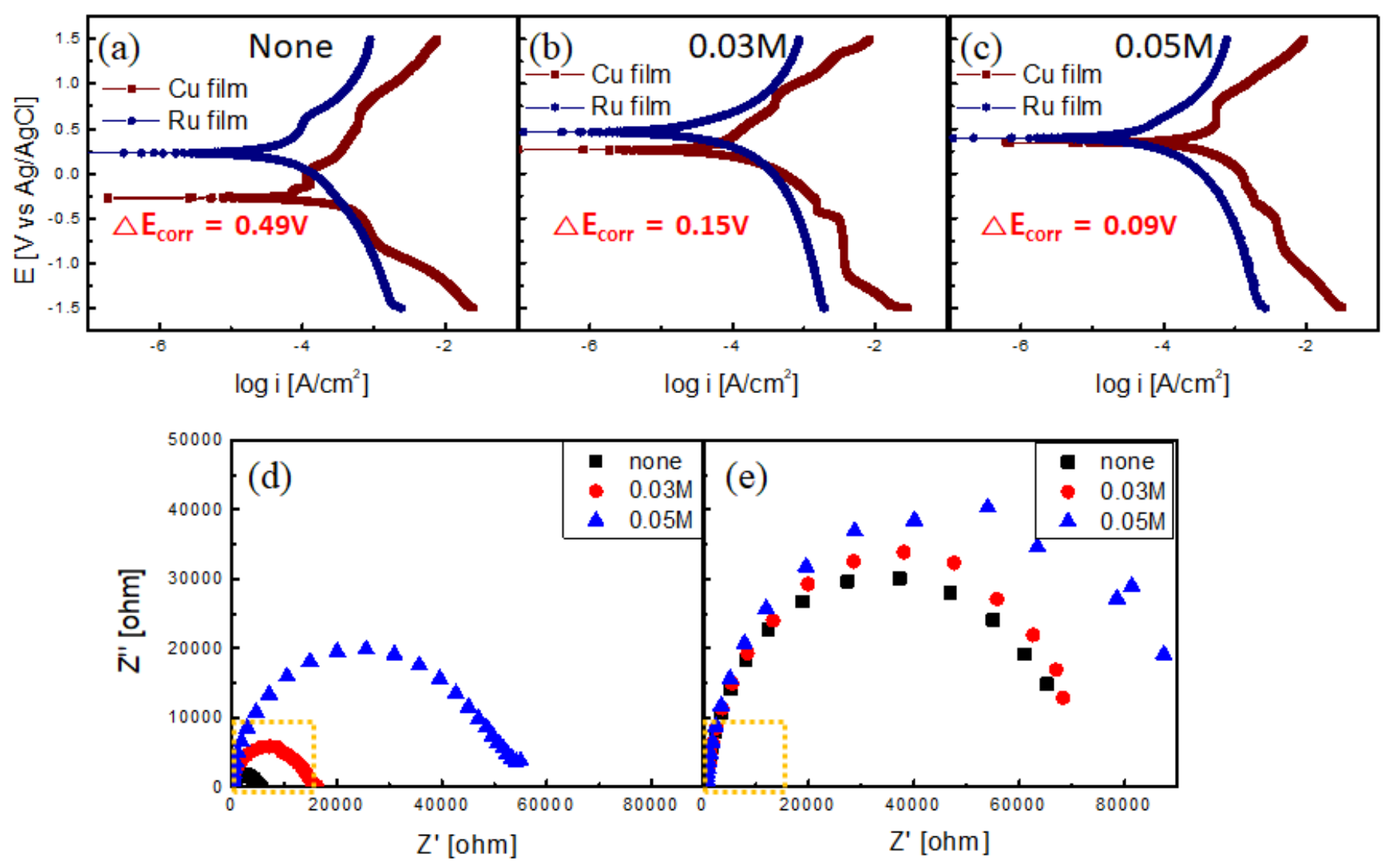

Figure 4

Electrochemical interaction evaluation between $\mathrm{Cu} / \mathrm{Ru}$ films and nicotinic acid inhibitor. Potentiodynamic plots for $\mathrm{Cu}$ and $\mathrm{Ru}$ in solution as a function of inhibitor concentrations at pH 10 (a-c): (a) w/o nicotinic acid, (b) 0.03M nicotinic acid, and (c) 0.05M nicotinic acid. Electrochemical impedance spectroscopy (EIS) data are showing Nyquist plots for (d) Cu film and (e) Ru film in solution for various inhibitor concentrations at $\mathrm{pH} 10$. 
(a)

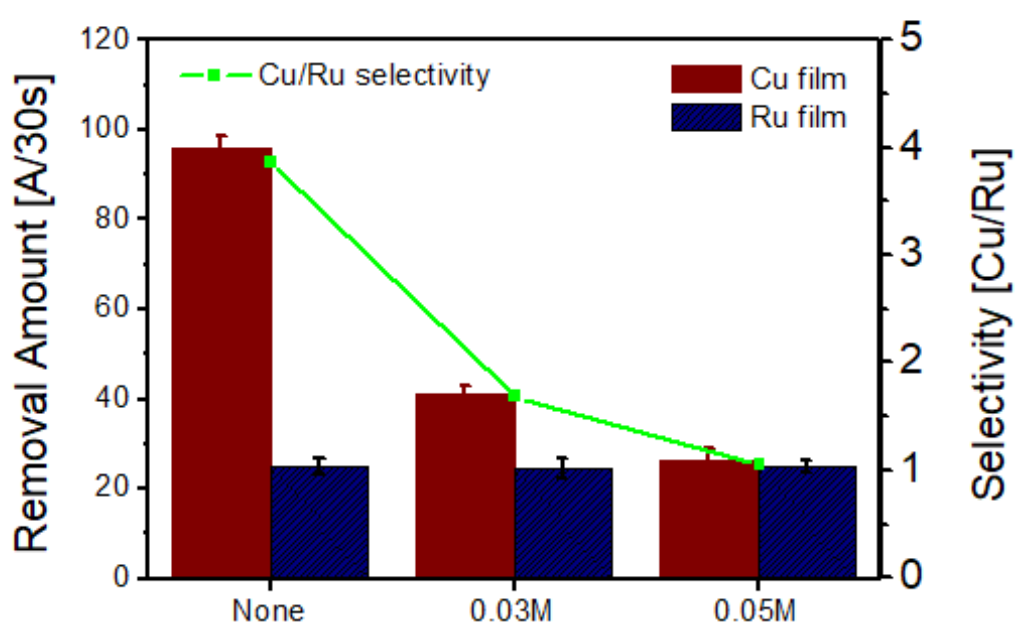

Concentration of Nicotinic acid [M]

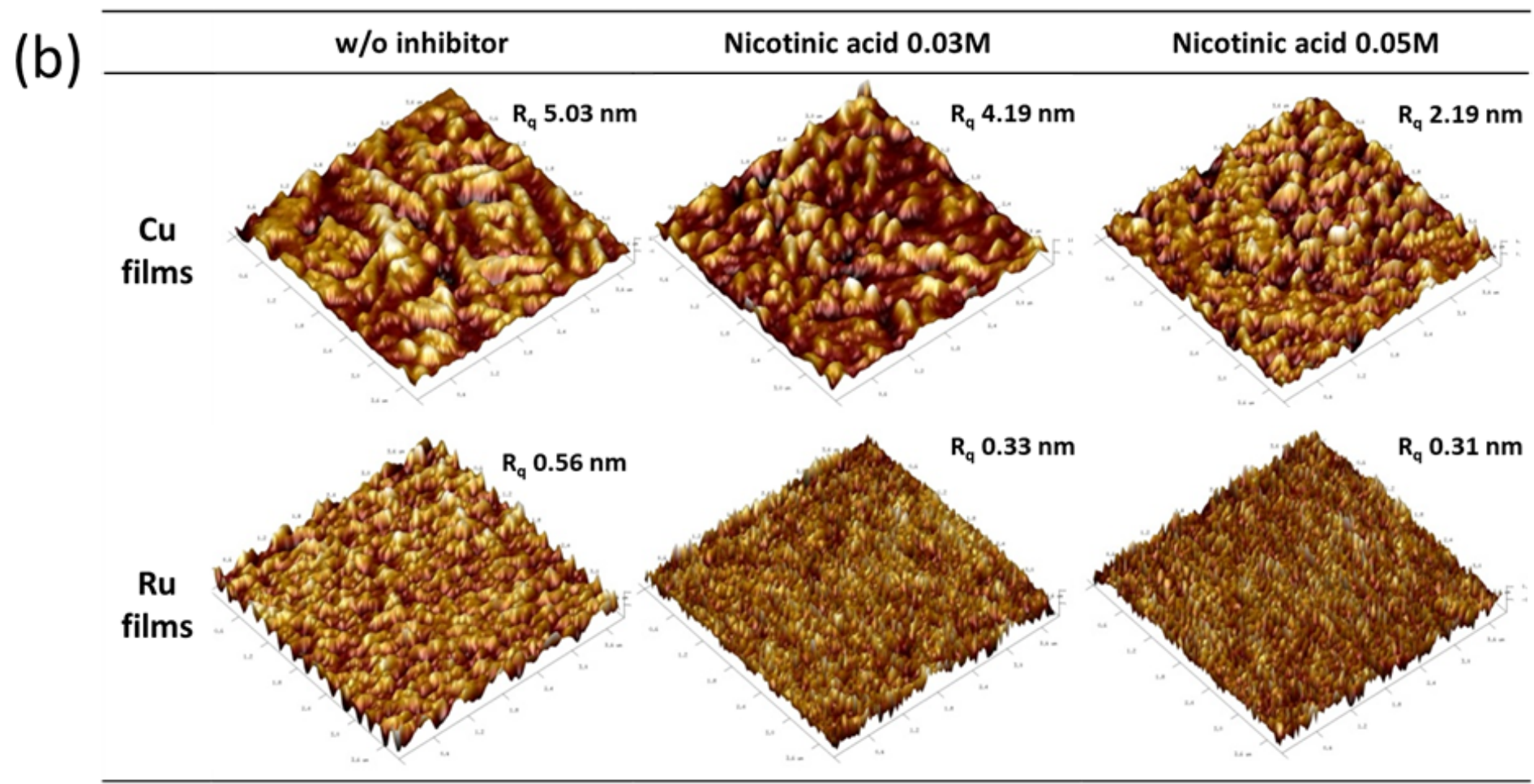

Figure 5

CMP performance for $\mathrm{Cu}$ and Ru films as a function of inhibitor concentrations at $\mathrm{pH} 10$. (a) The removal rate of the metal film via nicotinic acid concentration. (b) The surface morphologies of metal films on the surface under different nicotinic acid concentrations through AFM measurement.

\section{Supplementary Files}

This is a list of supplementary files associated with this preprint. Click to download. 